Bull. Austral. Math. Soc.

VOL. 41 (1990) [301-305]

\title{
ON NUNOKAWA'S CONJECTURE FOR MULTIVALENT FUNCTIONS
}

\section{SHIGEYOSHI OWA}

The object of this paper is to prove a conjecture given recently by Nunokawa that if $f(z) \in \mathcal{A}(p)$ satisfies $\operatorname{Re}\left\{1+z f^{\prime \prime}(z) / f^{\prime}(z)\right\}<p+1 / 2$ in the unit disk $U$, then $f(z)$ is p-valently starlike in $U$.

\section{INTRODUCTION}

Let $\mathcal{A}(p)$ denote the class of functions of the form

$$
f(z)=z^{p}+\sum_{n=p+1}^{\infty} a_{n} z^{n} \quad(n \in \mathcal{N}=\{1,2,3, \ldots\})
$$

which are analytic in the unit disk $\mathcal{U}=\{z:|z|<1\}$. A function $f(z)$ belonging to the class $\mathcal{A}(p)$ is said to be p-valently starlike in the unit disk $\mathcal{U}$ if it satisfies

$$
\operatorname{Re}\left\{\frac{z f^{\prime}(z)}{f(z)}\right\}>0
$$

for all $z \in \mathcal{U}$.

For functions $f(z) \in \mathcal{A}(1)$ when $p=1$, Singh and Singh [4] have proved

THEOREM A. If $f(z) \in \mathcal{A}(1)$ satisfies

$$
\operatorname{Re}\left\{1+\frac{z f^{\prime \prime}(z)}{f^{\prime}(z)}\right\}<\frac{3}{2} \quad(z \in \mathcal{U})
$$

then $f(z)$ is starlike in $\mathcal{U}$.

Recently, Nunokawa [3] has shown that

Theorem B. If $f(z) \in \mathcal{A}(p)$ satisfies

$$
\operatorname{Re}\left\{1+\frac{z f^{\prime \prime}(z)}{f^{\prime}(z)}\right\}<p+\frac{1}{4} \quad(z \in \mathcal{U})
$$

then $f(z)$ is p-valently starlike in $\mathcal{U}$.

In view of Theorem $A$ and Theorem $B$, Nunokawa [3] made the

\section{Received 2 May 1989}

Copyright Clearance Centre, Inc. Serial-fee code: 0004-9729/90 \$A2.00+0.00. 
Conjecture. If $f(z) \in \mathcal{A}(p)$ satisfies

$$
\operatorname{Re}\left\{1+\frac{z f^{\prime \prime}(z)}{f^{\prime}(z)}\right\}<p+\frac{1}{2} \quad(z \in \mathcal{U})
$$

then $f(z)$ is $p$-valently starlike in $\mathcal{U}$.

We note that this is true for $p=1$ by Theorem $A$. In this paper, we prove this conjecture for general $p$.

\section{Main Result}

For an analytic function $f(z)$ in $\mathcal{U}$, if $g(z)$ is univalent in $\mathcal{U}, f(0)=g(0)$, and $f(\mathcal{U}) \subseteq g(\mathcal{U})$, then $f(z)$ is said to be subordinate to $g(z)$. We denote this subordination by $f(z) \prec g(z)$.

In order to give our main result, we have to recall here the following lemma due to Jack [1] (see also Miller and Mocanu [2]).

Lemma 1. Let $w(z)$ be regular in $\mathcal{U}$ and such that $w(0)=0$. Then if $|w(z)|$ attains its maximum value on the circle $|z|=r$ at a point $z_{0} \in \mathcal{U}$, we have

$$
z_{0} w^{\prime}\left(z_{0}\right)=k w\left(z_{0}\right)
$$

where $k \geqslant 1$ is a real number.

Applying the above lemma, we derive

THEOREM 1. If $f(z) \in \mathcal{A}(p)$ satisfies the condition (1.5), then

$$
0<\operatorname{Re}\left\{\frac{z f^{\prime}(z)}{f(z)}\right\}<\frac{2 p(p+1)}{2 p+1} \quad(z \in \mathcal{U}) .
$$

Therefore, $f(z)$ is p-valently starlike in $U$. The result is sharp.

Proof: We define the function $w(z)$ by

$$
\frac{z f^{\prime}(z)}{f(z)}=\frac{p(p+1)(1-w(z))}{(p+1)-p w(z)} .
$$

Then $w(z)$ is regular in $\mathcal{U}$ and $w(0)=0$. It follows from (2.3) that

$$
1+\frac{z f^{\prime \prime}(z)}{f^{\prime}(z)}=\frac{p(p+1)(1-w(z))}{(p+1)-p w(z)}+\frac{p z w^{\prime}(z)}{(p+1)-p w(z)}+\frac{z w^{\prime}(z)}{1-w(z)}
$$

Suppose that there exists a point $z_{0} \in \mathcal{U}$ such that

$$
\max _{|z| \leqslant\left|z_{0}\right|}|w(z)|=\left|w\left(z_{0}\right)\right|=1
$$


Then, using Lemma 1, we have

$$
z_{0} w^{\prime}\left(z_{0}\right)=k w\left(z_{0}\right) \quad(k \text { is real and } k \geqslant 1),
$$

and $w\left(z_{0}\right)=e^{i \theta}$. Thus we obtain that

$$
\begin{aligned}
& \operatorname{Re}\left\{1+\frac{z_{0} f^{\prime \prime}\left(z_{0}\right)}{f^{\prime}\left(z_{0}\right)}\right\} \\
= & \operatorname{Re}\left\{\frac{p(p+1)\left(1-w\left(z_{0}\right)\right)}{(p+1)-p w\left(z_{0}\right)}+\frac{p k w\left(z_{0}\right)}{(p+1)-p w\left(z_{0}\right)}-\frac{k w\left(z_{0}\right)}{1-w\left(z_{0}\right)}\right\} \\
= & \frac{p(p+1)(2 p+1)(1-\cos \theta)+p k((p+1) \cos \theta-p)}{\left(2 p^{2}+2 p+1\right)-2 p(p+1) \cos \theta}+\frac{k}{2} \\
= & p+\frac{1}{2}+\frac{(2 p+1)(k-1)}{\left(2 p^{2}+2 p+1\right)-2 p(p+1) \cos \theta} \\
\geqslant & p+\frac{1}{2}
\end{aligned}
$$

which contradicts our condition (1.5). Hence, we conclude that $|w(z)|<1$ for all $z \in \mathcal{U}$. Further, noting that the function $g(z)$ defined by

$$
g(z)=\frac{p(p+1)(1-z)}{(p+1)-p z}
$$

is univalent in $\mathcal{U}$ and $g(0)=p$, we have that

$$
\frac{z f^{\prime}(z)}{f(z)} \prec g(z) \quad(z \in \mathcal{U}),
$$

that is, that

$$
\begin{aligned}
\operatorname{Re}\left\{\frac{z f^{\prime}(z)}{f(z)}\right\} & \geqslant \min _{|z|=r} \operatorname{Re} g(z) \\
& =\frac{p(p+1)(1-r)}{(p+1)-p r} \\
& >0 \quad(|z|=r<1)
\end{aligned}
$$

and

$$
\begin{aligned}
\operatorname{Re}\left\{\frac{z f^{\prime}(z)}{f(z)}\right\} & \leqslant \max _{|z|=r} \operatorname{Re}\{g(z)\} \\
& =\frac{p(p+1)(1+r)}{(p+1)+p r} \\
& <\frac{2 p(p+1)}{2 p+1} \quad(|z|=r<1)
\end{aligned}
$$


Further, we see that the result is sharp for the function

$$
f(z)=z^{p}\{(p+1)-p z\} .
$$

This completes the proof.

Making $p=1$ in Theorem 1, we have

Corollary 1. If $f(z) \in \mathcal{A}(1)$ satisfies

then

$$
\operatorname{Re}\left\{1+\frac{z f^{\prime \prime}(z)}{f^{\prime}(z)}\right\}<\frac{3}{2} \quad(z \in \mathcal{U}),
$$

$$
0<\operatorname{Re}\left\{\frac{z f^{\prime}(z)}{f(z)}\right\}<\frac{4}{3} \quad(z \in \mathcal{U}) .
$$

The result is sharp.

Next, we prove

THEOREM 2. If $f(z) \in \mathcal{A}(p)$ satisfies

$$
\operatorname{Re}\left\{1+\frac{z f^{\prime \prime}(z)}{f^{\prime}(z)}\right\}>\frac{p}{4}-1 \quad(z \in \mathcal{U}),
$$

then

$$
\operatorname{Re} \sqrt{\frac{z f^{\prime}(z)}{f(z)}}>\frac{p^{1 / 2}}{2} \quad(z \in \mathcal{U})
$$

The result is sharp.

PRoof: Defining the function $w(z)$ by

$$
\sqrt{\frac{z f^{\prime}(z)}{f(z)}}=\frac{p^{1 / 2}}{1+w(z)},
$$

we have

$$
1+\frac{z f^{\prime \prime}(z)}{f^{\prime}(z)}=\frac{p}{(1+w(z))^{2}}-\frac{2 z w^{\prime}(z)}{1+w(z)}
$$

Assuming that there exists a point $z_{0} \in \mathcal{U}$ such that

$$
\max _{|z| \leqslant\left|z_{0}\right|}|w(z)|=\left|w\left(z_{0}\right)\right|=1,
$$


Lemma 1 implies that

$$
\begin{aligned}
\operatorname{Re}\left\{1+\frac{z_{0} f^{\prime \prime}\left(z_{0}\right)}{f^{\prime}\left(z_{0}\right)}\right\} & =\operatorname{Re}\left\{\frac{p}{\left(1+w\left(z_{0}\right)\right)^{2}}-\frac{2 k w\left(z_{0}\right)}{1+w\left(z_{0}\right)}\right\} \\
& =\frac{p \cos \theta}{2(1+\cos \theta)}-k \\
& \leqslant \frac{p}{4}-1,
\end{aligned}
$$

where $w\left(z_{0}\right)=e^{i \theta}$. This proves that

$$
\operatorname{Re} \sqrt{\frac{z f^{\prime}(z)}{f(z)}}=\operatorname{Re}\left\{\frac{p}{1+w(z)}\right\}>\frac{p}{2} \quad(z \in \mathcal{U}) .
$$

Noting that $g(z)=p^{1 / 2} /(1+z)$ is univalent in $U$ and $g(0)=p^{1 / 2}$, so that

$$
\sqrt{\frac{z f^{\prime}(z)}{f(z)}} \prec g(z)=\frac{p^{1 / 2}}{1+z},
$$

we see that the result is sharp with the extremal function

$$
f(z)=\left(\frac{z}{1+z} e^{z /(1+z)}\right)^{p}
$$

Setting $p=1$ in Theorem 2, we have

CoRollary 2. If $f(z) \in \mathcal{A}(1)$ satisfies

then

$$
\operatorname{Re}\left\{1+\frac{z f^{\prime \prime}(z)}{f^{\prime}(z)}\right\}>-\frac{3}{4} \quad(z \in \mathcal{U})
$$

$$
\operatorname{Re} \sqrt{\frac{z f^{\prime}(z)}{f(z)}}>\frac{1}{2} \quad(z \in \mathcal{U}) .
$$

\section{REFERENCES}

[1] I.S. Jack, 'Functions starlike and convex of order $\alpha$ ', J. London Math. Soc. 3 (1971), 469-474.

[2] S.S. Miller and P.T. Mocanu, 'Second order differential inequalities in the complex plane", J. Math. Anal. Appl. 65 (1978), 289-305.

[3] M. Nunokawa, 'On the multivalent functions', Indian J. Pure Appl. Math. 20 (1989), 577-582.

[4] R. Singh and S. Singh, 'Some sufficient conditions for univalence and starlikeness', Colloq. Math. 47 (1982), 309-314.

Department of Mathematics

Kinki University

Higashi-Osaka, Osaka 577

Japan 
